\title{
Time Dependent Adaptive Configuration Interaction Applied to Attosecond Charge Migration
}

\author{
Jeffrey B. Schriber ${ }^{1}$ and Francesco A. Evangelista ${ }^{2}$ \\ ${ }^{1)}$ Center for Computational Molecular Science and Technology, School of Chemistry and Biochemistry, \\ Georgia Institute of Technology, Atlanta, Georgia, 30318, USA a) \\ 2) Department of Chemistry and Cherry L. Emerson Center for Scientific Computation, Emory University, Atlanta, Georgia, 30322, \\ USA
}

(Dated: 18 September 2019)

In this work, we present a time-dependent (TD) selected configuration interaction method based on our recentlyintroduced adaptive configuration interaction (ACI). We show that ACI, in either its ground or excited state formalisms, is capable of building a compact basis for use in real-time propagation of wave functions for computing electron dynamics. TD-ACI uses an iteratively selected basis of determinants in real-time propagation capable of capturing strong correlation effects in both ground and excited states, all with an accuracy—and associated cost—-tunable by the user. We apply TD-ACI to study attosecond-scale migration of charge following ionization in small molecules. We first compute attosecond charge dynamics in a benzene model to benchmark and understand the utility of TD-ACI with respect to an exact solution. Finally, we use TD-ACI to reproduce experimentally determined ultrafast charge migration dynamics in iodoacetylene. TD-ACI is shown to be a valuable benchmark theory for electron dynamics, and it represents an important step towards accurate and affordable time-dependent multireference methods.

Recent developments of high-intensity attosecond laser pulses 1 can provide valuable insight to the phenomena of charge migration, defined as the electronic motion, e.g. following ionization, that occurs before a nuclear response. $2+5$ Charge migration has been used to explain site-selective reactivity in electronically excited peptides $\frac{67}{7}$ and can potentially be used to direct chemical reactions into normally inaccessible pathways. While initial work on experimental measurement and control of charge migration is promising,, 8 theoretical techniques are required to understand specific electron dynamics pathways and to begin answering questions on the greater feasibility of charge migration controlled chemistry.

Cederbaum and Zoberly first introduced the idea that electron correlation in populated excited cationic states drives attosecond charge migration following ionization. Following this work, numerous studies using the nonDyson intermediate-state representation of the third or fourth order algebraic diagrammatic construction [ADC(3), $\mathrm{ADC}(4)]$, 5[14 23 time-dependent density functional theory (TD-DFT), 24 26 and time-dependent density matrix renormalization group (TD-DMRG) $)^{27 / 28}$ have been employed to further understand pure electron dynamics following ionization. The major theoretical challenge of describing coherent electron dynamics following ionization is in accurately characterizing the populated cationic states. $\operatorname{ADC}(3), \operatorname{ADC}(4)$, and TD-DFT are affordable options to study charge migration, but they inherently depend on a single-reference description of correlation effects. Failure to characterize strong correlation in excited states can lead to a qualitatively incorrect description of the dynamics and is likely to occur when numerous near-degenerate cationic states with many coupled excitations become populated. TD-DMRG can treat an electronic state beyond the traditional excitation level hierarchy and is thus

\footnotetext{
a)Electronic mail: jschriber7@ gatech.edu

b)Electronic mail: francesco.evangelista@emory.edu
}

well-positioned for computing complicated dynamics, despite challenges associated with excited-state DMRG algorithms and propagating a matrix product state wave function. 27,30

In this work, we generalize the adaptive configuration interaction 31 method (ACI) to a time-dependent version (TD-ACI) for simulating electron dynamics. ACI is based on the very old idea of selected $\mathrm{Cl}^{34} 48$ and is one of numerous new manifestations of these techniques! 69 TD-ACI can be viewed as an approximation to time-dependent complete active space (CAS) techniques, which have been successfully applied to photoionization processes,, 68 where determinant screening in ACI enables the use of much larger CAS spaces. Due to its systematic improvability and demonstrated ability to treat many strongly correlated electrons in ground and excited states, TD-ACI will be essential for benchmarking time dependent methods and for providing insight to the role of electron correlation in attosecond electron dynamics.

Studying ultrafast dynamics of electrons requires the solution to the time-dependent Schrödinger equation (TDSE), in atomic units, $i \frac{\partial}{\partial t}|\Psi(t)\rangle=\hat{H}|\Psi(t)\rangle$, which dictates how a wave function, $\Psi(t)$, evolves in time according to the Hamiltonian $(\hat{H})$. For a time-independent $\hat{H}$, the evolution of a wave function can be written exactly,

$$
|\Psi(t+\Delta t)\rangle=e^{-i \hat{H} \Delta t}|\Psi(t)\rangle
$$

where $\Delta t$ is the timestep over which the wave function evolves. To avoid the combinatorial complexity of the exact full configuration interaction (FCI) solution to the TDSE, many approximate time-dependent methods in electronic structure theory have been proposed to study pure electron dynamics, including real-time versions of Hartree-Fock (HF) theory, 6970 configuration interaction, 67/71-76 multiconfigurational self consistent field wave functions, ${ }^{77+79}$ density functional theory, 24$] 80] 82$ and coupled-cluster theories ${ }^{83}$ To study charge migration following ionization, equation 1 needs to be accurately approximated by $(i)$ generating an accurate initial ionized state, (ii) building a tractably-sized cationic 

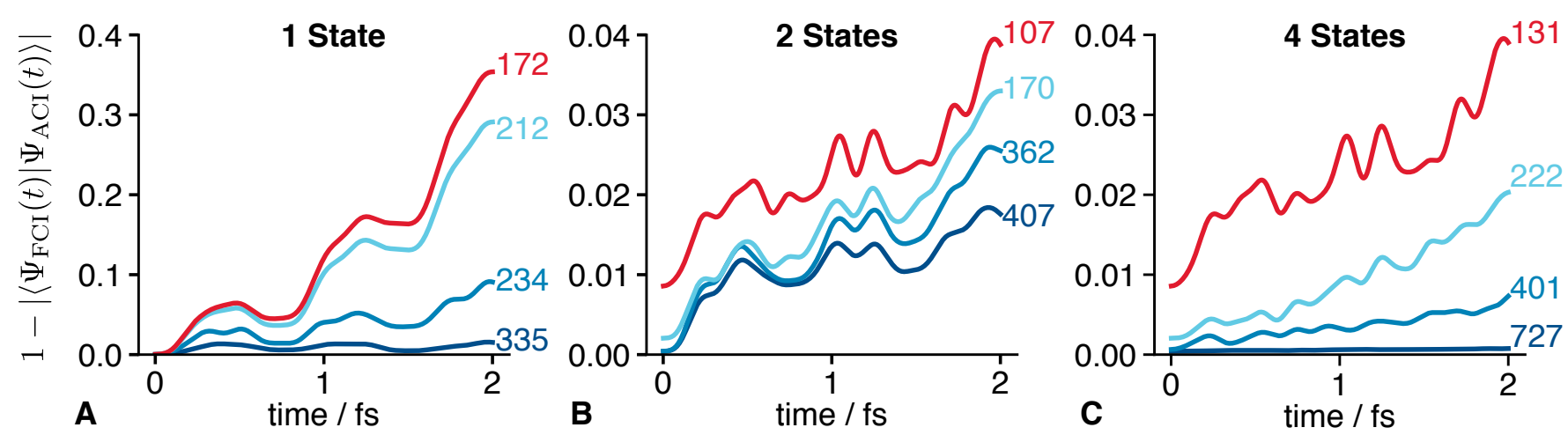

FIG. 1. Errors in the overlap between TD-ACI wave functions and the exact wave function, for which $\left|M^{N-1}\right|=3920$ determinants. TD-ACI wave functions are computed with various $\sigma$ values using one $(\mathbf{A})$, two $(\mathbf{B})$, or four $(\mathbf{C})$ reference states in computing the cationic basis. Each error curve is labeled with the number of determinants in the cationic space. The FCI wave functions are propagated exactly, and TD-ACI wave functions are propagated using RK4 with a timestep of 0.05 as.

Hamiltonian that well-describes all populated cationic states throughout propagation, and (iii) avoiding evaluation of the exponential using conventional numerical integrators.

We use ACI to build a compact determinantal representation of $\hat{H}$ and a well-defined initial ionized state. ACI uses an iterative screening algorithm to build a model space, $M^{N}$, composed of $N$-electron Slater determinants $\left(\Phi^{N}\right)$, such that the resultant wave function, $\left|\Psi^{N}\right\rangle=\sum_{\Phi_{\mu}^{N} \in M^{N}}\left|\Phi_{\mu}^{N}\right\rangle c_{\mu}$, produces an energy error approximately equal to a user-defined parameter, $\sigma\left(\left|E_{\mathrm{ACI}}-E_{\mathrm{FCI}}\right| \approx \sigma\right) ![3]$ We have reported several algorithms to compute excited states with ACI, including a state-averaged ACI (SA-ACI) which optimizes a single model space by defining determinant importance as the contribution to the correlation energy averaged over several roots. ${ }^{32}$ This algorithm is very useful in formulating a time-dependent theory as it produces a compact Hamiltonian with a controllable average energy error over any number of roots.

To study charge migration following ionization, we first perform a SA-ACI computation on the lowest one or few states of the neutral molecule of interest to build the ground state wave function, $\left|\Psi_{0}^{N}\right\rangle$. We invoke the sudden ionization approximation, $\frac{59127}{5}$ were the initial wave function for the simulation $\left[\Psi^{N-1}(t=0)\right]$ is defined immediately after the ionization process by annihilating an electron in spin orbital $\phi_{i}$ and normalized appropriately as

$$
\left|\Psi^{N-1}(t=0)\right\rangle=\frac{\hat{a}_{i}\left|\Psi_{0}^{N}\right\rangle}{\left\langle\Psi_{0}^{N}\left|\hat{a}_{i}^{\dagger} \hat{a}_{i}\right| \Psi_{0}^{N}\right\rangle} .
$$

The sudden ionization approximation provides a well-defined initial state suitable for benchmarking the propagation in TDACI field-free with a time-independent Hamiltonian. In practice, TD-ACI simulations can include the ground electronic state interacting with an ionizing pulse, but invoking the sudden ionization approximation allows us to effectively decouple errors associated with TD-ACI and those connected to our choice of initial conditions. Since ACI uses a linear expansion of Slater determinants, the initial state can be defined by either a localized hole or a superposition of holes without complications possible in other approaches. 2688889
We then define the basis for the cationic Hamiltonian, $M^{N-1}$, as the set of cationic determinants, $\left\{\Phi^{N-1}\right\}$, generated by applying a single annihilation over all spin orbitals to all determinants in the original basis $M^{N}, M^{N-1}=\left\{\Phi_{I}^{N-1}\right.$ : $\Phi_{I}^{N-1}=\hat{a}_{i}\left|\Phi_{\mu}^{N}\right\rangle, \forall i \in \mathbf{A}$ and $\left.\forall \Phi_{\mu}^{N} \in M^{N}\right\}$, where $\mathbf{A}$ denotes the set of all active orbitals. Lastly, we define the TD-ACI wave function with the time-dependence entirely encoded in the expansion coefficients $\left[c_{I}(t)\right]$ as $\left|\Psi^{N-1}(t)\right\rangle=$ $\sum_{\Phi_{I}^{N-1} \in M^{N-1}} c_{I}(t)\left|\Phi_{I}^{N-1}\right\rangle$. Starting from the initial condition [equation [2]], the wave function $\Psi^{N-1}(t)$ can be determined at any time $t$ by integration of the TDSE. Exact propagation via equation 1 requires complete diagonalization of the cationic Hamiltonian and is unfeasible for realistic simulations. Instead, we use the fourth-order Runge-Kutta (RK4) algorithm with fixed timesteps, $27 / 30|90| 91$ which for a timeindependent $\hat{H}$ is equivalent to approximating the exact exponential propagator using a Taylor series truncated to fourth order. We monitor the migration of the ionized hole throughout the molecule using the hole occupation number, $n_{i}$ for an orbital $i$, defined as the difference between the occupation of orbital $i$ in the ground state and the ionized state at time $t$, $n_{i}(t)=\left\langle\Psi_{0}^{N}\left|\hat{a}_{i}^{\dagger} \hat{a}_{i}\right| \Psi_{0}^{N}\right\rangle-\left\langle\Psi^{N-1}(t)\left|\hat{a}_{i}^{\dagger} \hat{a}_{i}\right| \Psi^{N-1}(t)\right\rangle$.

We first study dynamics triggered by valence ionization in benzene to understand the effects of using a truncated cationic model space and an approximate time propagator. The dynamics of the ionized state is characterized by hole migration within the $\pi / \pi^{*}$ manifold, with weak hole-occupation of a nearby $\sigma$-bonding orbital. The benzene geometry was optimized using DFT with a B3LYP functional and the cc-pVDZ basis set using the PSI4 program. ${ }^{92]} \mathrm{ACI}$ computations use a $\operatorname{CAS}(8,8)$ containing the $\pi / \pi^{*}$ valence space and the energetically nearest $\sigma / \sigma^{*}$ bonding/antibonding pair, and they employ a restricted HF reference computed with the cc-pVDZ basis and density fitted integrals using the cc-pVDZ-JKFIT basis. ${ }^{93}$ The computation was run using $C_{1}$ symmetry, but we refer to using corresponding $D_{2 h}$ labels for clarity, all plotted in figure S1. In the TD-ACI simulation, we use a time step of 0.05 as for a total time of $2 \mathrm{fs}$, and the initial state is prepared by annihilating the alpha $1 b_{1 u}$ spin orbital. 
Upon ionization of the $1 b_{1 u}$ orbital, the hole migrates to a superposition of the degenerate $1 b_{2 g}$ and $1 b_{3 g}$ orbitals and back smoothly with a frequency of roughly 750 as. The oscillation is faster by about 200 as compared to previously reported ADC(3) results because of the minimal CAS $(8,8)$ employed in this work ${ }^{[22}$ This reduced model is nonetheless an effective test of our theory because the oscillating hole occupations require determinants different in character from those that define the ground cationic state. We test three different schemes to build $M^{N-1}$ from a SA-ACI computation of $M^{N}$ optimized with respect to one, two, or four roots of the neutral species. These time-independent computations are run with values of $\sigma$ chosen to produce similar dimensions of $M^{N-1}$ to facilitate comparisons.

With the various $M^{N-1}$ bases, we propagate the initial wave functions using the RK4 algorithm with a 0.05 as timestep, and we plot the error in their overlaps with respect to the FCI result in Figure 1. Due to the short timestep used, the observed errors are largely resultant from truncation alone, and we see it is not necessarily correlated with the number of determinants in each computation. For example, the propagations with $M^{N-1}$ generated from a single state 1 A) show errors in the overlap up to 0.3 at 2 fs when the cationic space contains about 200 determinants. With a similar dimension, the twostate variant already shows an approximate 10-fold increase in accuracy. This increase in accuracy is due to the influence of two-hole/one-particle states and simple hole-excited states in the hole dynamics. ${ }^{[2]}$ To describe all of these states, $M^{N-1}$ needs determinants with single, double, or higher excitation character with respect to ionized ground and excited states. Our results indicate that such a determinantal makeup is most effectively achieved by ionizing determinants from an SAACI computation done with respect to several excited states.

The hole migration from the $1 b_{1 u}$ orbital to a superposition of $1 b_{2 g}$ and $1 b_{3 g}$ orbitals is also shown in Figure 2. In the left plots, we show hole occupations computed with TD-ACI using the exact propagator with either one $(2, \mathbf{A})$ or four $2, \mathbf{B})$ optimized roots in defining $M^{N-1}$. The plots on the right show the same data, but instead using the RK4 propagator. Our first observation is that the TD-ACI dynamics are indistinguishable when using either exact or RK4 propagatiors, showing that our expected propagation errors are negligible even with determinant selection so long as an appropriate time step is chosen. When the $M^{N-1}$ is optimized with respect to one root, the hole occupation oscillates too quickly and does not transfer from the $1 b_{1 u}$ orbital (red) to the $1 b_{2 g}$ and $1 b_{3 g}$ orbitals (blue and green) with enough magnitude. Interestingly, the hole occupations of the $1 b_{2 g}$ and $1 b_{3 g}$ orbitals are correctly always identical. The four-state procedure to build a similarlysized $M^{N-1}$ gives occupations nearly indistinguishable from the exact result. These results indicate that TD-ACI is a viable technique in studying charge migration resultant from ionization, particularly if the basis for the cationic Hamiltonian is truncated using importance criteria that can consider multiple roots. This use of an SA-ACI computation to build the basis enables a faster convergence to the $\sigma=0$ limit, and does not bias the determinantal makeup of cationic Hamiltonian towards the ground cationic state.

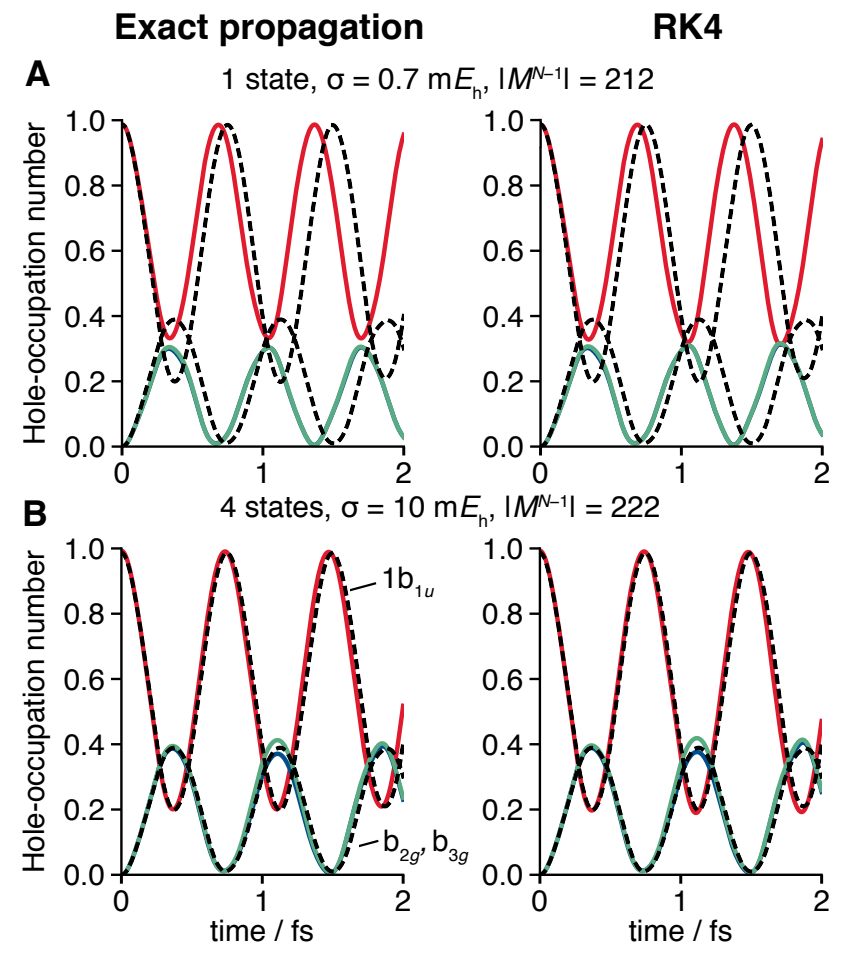

FIG. 2. Hole occupations of $1 b_{1 u}, 1 b_{2 g}$, and $1 b_{3 g}$ orbitals computed using TD-ACI with $M^{N-1}$ optimized for 1 (A) and 4 (B) states using exact propagation (left) and RK4 propagation (right) with a 0.05 as time step. TD-FCI occupations are shown withe a dashed grey line.

Iodoacetylene is a valuable model of charge migration for theorists and experimentalists alike. Recently, Kraus et. al have used high-harmonic generation to achieve 100 as resolution and control over charge migration following ionization. The dynamics following ionization was also investigated theoretically in a TD-DMRG study using a CAS $(16,36)$ active space. ${ }^{27}$ In iodoacetylene, two cationic states drive the dynamics, one characterized by a hole in the $5 p$-like orbitals of iodine perpendicular to the molecular axis, and the other having a hole in the two $\pi$-bonding orbitals in the acetylene triple bond. These states are near degenerate and a multiconfigurational time-dependent approach may be required. The dynamics of ionized iodoacetylene involve the hole migrating between the iodine and acetylene groups with an experimentally measured frequency of about 0.93 fs $[4 \mid 8$ Our final test of TD-ACI is to reproduce this migration frequency using a truncated basis of cationic determinants.

We optimize the geometry with DFT using a PBEC 94 functional and def2-SVP basis set. ${ }^{95}$ This basis was used in all iodoacetylene computations in addition to an effective core potential used to remove 28 core electrons. Within the remaining 59 orbitals, we use a CAS $(16,22)$ for the neutral species, which includes all molecular orbitals generated from the $2 p$ like orbitals in the acetylene group, and $4 d$ and $5 p$-like molecular orbitals from the iodine. In the dynamics simulations, we use a timestep of 0.02 as for a total simulation time of $2 \mathrm{fs}$. The initial state is prepared by annihilating a valence $5 p$-like orbital on the iodine atom, and all computations are run in $C_{1}$ 


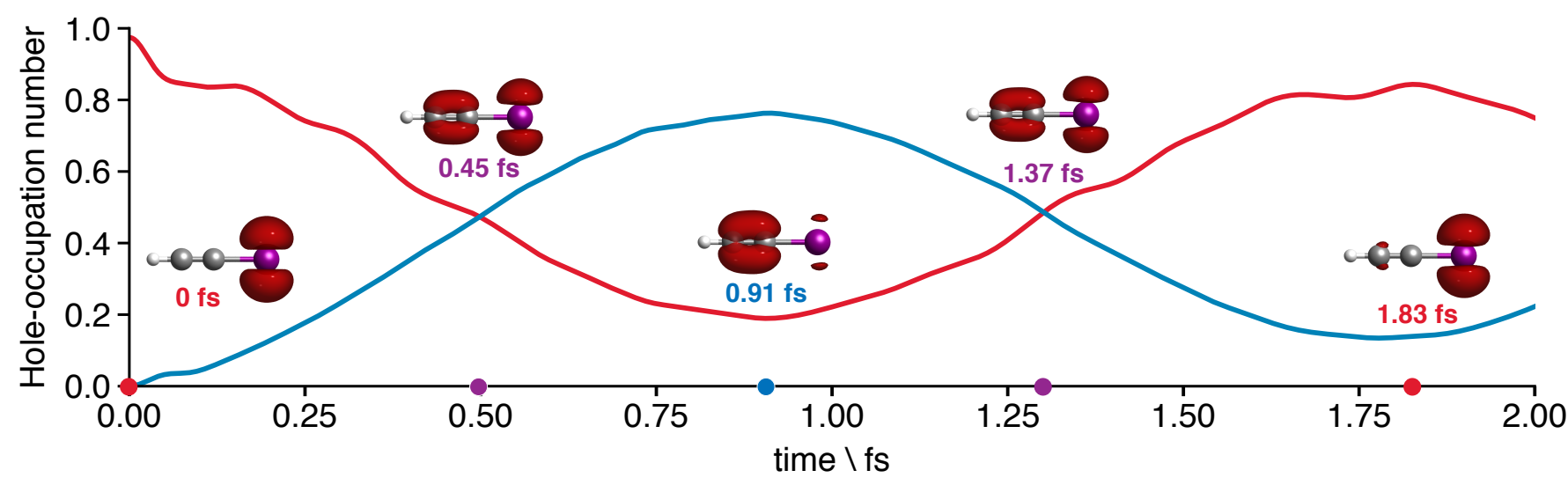

FIG. 3. Hole occupations of the $5 p$-like orbital on iodine (red) and the $\pi$ orbital on the acetylene (blue) in the same plane. Evolution of the hole density $\left(\rho_{\mathrm{h}}\right)$, as defined in the text, is shown at maxima, minima and intersections of the hole occupation curves.

symmetry with split-localized orbitals in the active space.

To analyze the charge migration dynamics, we compute a representation of the density of the ionized hole $\left(\rho_{\mathrm{h}}\right)$ by scaling the HF orbitals by the corresponding hole occupation,, 927 $\rho_{\mathrm{h}}=\sum_{i}\left|\phi_{i}\right|^{2} n_{i}$. Our first simulation uses a two-state SA-ACI computation with $\sigma=10 m E_{\mathrm{h}}$ to generate a cationic basis containing 244,361 determinants, where the cationic Hilbert space contains roughly $5 \times 10^{10}$ determinants. We plot the hole occupation numbers for the ionized $5 p$-like orbital on the iodine and the in-plane $\pi$-bonding orbital of the acetylene in Figure 3 for the 2 fs simulation. The hole migrates from the iodine to the acetylene and back in about $0.91 \mathrm{fs}$, agreeing closely with the experimental frequency of $0.93 \mathrm{fs}$. The migration still leaves some degree of hole occupation on each moiety, which we show Figure 3 . The relatively good agreement between our computed frequency of hole migration and the experimental value suggests that the ACI procedure is building an adequate space of determinants to describe the relevant cationic states.

To test the sensitivity of the dynamics with respect to $M^{N-1}$, we show hole migration times with TD-ACI using cationic spaces built from one, two and three root SA-ACI computations in Table T. The acetylene migration time is defined as the time required for the ionized hole, initially on the iodine, to maximally populate on the acetylene group. The iodine migration time is the time elapsed from initial ionization to repopulation of the hole density on iodine after it has migrated. For a nearly constant size of $M^{N-1}$, we see that increasing the number of states used to generate the cationic basis has a negligible effect on the hole migration times. Only when the total number of cationic determinants is increased, even when optimized for the neutral ground state, do we see the migration times approach the experimental and TD-DMRG values. This result depends solely on the determinantal makeup of the cationic states most important in forming the evolving wave packet. For iodoacetylene, these states are simple 1-hole cationic states with the hole located on the iodine or acetylene moieties, and no coupled electronic excitations are as significant as they were for our previous study on benzene. As a result, the dominant contribution for both hole states comes
TABLE I. Iodoacetylene hole migration times computed with TDACI using various $M^{N-1}$, and from experiment (Exp.) from Ref. 8

\begin{tabular}{cccc}
\hline & & \multicolumn{2}{c}{ Migration Time (as) } \\
\cline { 3 - 4 } Number of States & $\left|M^{N-1}\right|$ & Acetylene & Iodine \\
\hline 1 & 10127 & 862 & 1684 \\
2 & 10827 & 864 & 1660 \\
3 & 10759 & 868 & 1610 \\
1 & 93554 & 886 & 1780 \\
2 & 244361 & 907 & 1828 \\
Exp. & & 930 & 1850 \\
\hline
\end{tabular}

from different annihilations of the ground state and not from annihilations of excited states.

Our correlation treatment provides accurate dynamics despite the neglect of dynamical correlation. While fortuitous cancellation of error is possibly present and some dynamical correlation may be recovered within our active spaces, the success of the active-space treatment suggests that dynamical correlation is relatively unimportant in accurately defining the relevant cationic states for propagation. While dynamical correlation is likely necessary for accurately computing other time-dependent properties, TD-ACI shows great promise in computing time-dependent reference wave functions. A very appealing property of our TD-ACI approach is that we can effectively optimize $M^{N-1}$ regardless of the character of the relevant cationic states, whether simple or complex.

In this work, we have extended the ACI approach to simulate ultrafast electron dynamics. TD-ACI is able to effectively model the real-time propagation of the exact wave function using a truncated space of determinants selected by the time-independent ACI algorithm. We apply this methodology to charge migration dynamics that follow ultrafast ionization. We find that $\mathrm{ACI}$ is well-suited to find determinants relevant to the initial state, and determinants important at later points in time can be initially identified in excited state computations to reduce systematic increases in error as the wave function evolves. We also find that the single-state ACI computation can recover these determinants if a sufficiently large value of $\sigma$ is used. Due to the short time scale of charge migration, we did not encounter significant issues in using approximations 
to the exact time propagator, though these effects could be important in longer simulations. Propagation error and truncation errors were studied using a charge migration model in benzene, where we found that cationic spaces optimized for multiple roots are needed to efficiently capture dynamics in which two-hole one-particle states are relevant. This type of state is not always relevant to the dynamics in propagating our initial state, as we saw in our application to hole migration in iodoacetylene. Using various schemes to build our cationic basis, we were able to compute hole migration times between acetylene and iodine groups that match experimental results.

We anticipate numerous future directions of study. While the SA-ACI procedure was successful in building a determinantal space for the entire dynamics, we envision an even more efficient scheme where the selection and removal of determinants can be done during the simulation itself. For example, one can imagine an algorithm that estimates the importance of a determinant at a future timestep using loworder approximate propagation. Additionally, integration of the current TD-ACI scheme with a time-dependent Hamiltonian would enable $a b$ initio studies of molecules interacting with fluctuating electric or magnetic fields. Finally, we envision including dynamical correlation effects beyond our active space treatment by combining an effective Hamiltonian theory with our time-dependent reference wave functions.

\section{ACKNOWLEDGMENTS}

This work was supported by the U.S. National Science Foundation under Grant No. CHE 1900532 and a Camille Dreyfus Teacher-Scholar Award (TC-18-045). J.B.S. would like to thank Dr. Tianyuan Zhang for helpful discussions on approximate propagation techniques.

${ }^{1}$ F. Krausz and M. Ivanov, "Attosecond physics," Rev. Mod. Phys. 81, 163234 (2009).

${ }^{2}$ M. Nisoli, P. Decleva, F. Calegari, A. Palacios, and F. Martín, "Attosecond Electron Dynamics in Molecules." Chem. Rev. 117, 10760-10825 (2017).

${ }^{3}$ S. R. Leone, C. W. McCurdy, J. Burgdörfer, L. S. Cederbaum, Z. Chang, N. Dudovich, J. Feist, C. H. Greene, M. Ivanov, R. Kienberger, U. Keller, A. Stolow, U. Thumm, and M. J. J. Vrakking, "What will it take to observe processes in 'real time'?" Nat. Photon. 8, 162-166 (2014).

${ }^{4}$ P. M. Kraus and H. J. Wörner, "Perspectives of Attosecond Spectroscopy for the Understanding of Fundamental Electron Correlations." Angew. Chem. Int. Ed. 57, 5228-5247 (2018).

${ }^{5}$ V. Despré, N. V. Golubev, and A. I. Kuleff, "Charge Migration in Propiolic Acid: A Full Quantum Dynamical Study." Phys. Rev. Lett. 121, 203002 (2018).

${ }^{6}$ R. Weinkauf, P. Schanen, A. Metsala, E. W. Schlag, M. Bürgle, and H. Kessler, "Highly Efficient Charge Transfer in Peptide Cations in the Gas Phase: Threshold Effects and Mechanism," J. Phys. Chem. 100, 1856718585 (1996).

${ }^{7}$ R. Weinkauf, E. W. Schlag, T. J. Martinez, and R. D. Levine, "Nonstationary Electronic States and Site-Selective Reactivity," J. Phys. Chem. A 101, 7702-7710 (1997).

${ }^{8}$ P. M. Kraus, B. Mignolet, D. Baykusheva, A. Rupenyan, L. Horný, E. F. Penka, G. Grassi, O. I. Tolstikhin, J. Schneider, F. Jensen, L. B. Madsen, A. D. Bandrauk, F. Remacle, and H. J. Wörner, "Measurement and laser control of attosecond charge migration in ionized iodoacetylene," Science 350, 790-795 (2015).

${ }^{9}$ L. S. Cederbaum and J. Zobeley, "Ultrafast charge migration by electron correlation,” Chem. Phys. Lett. 307, 205-210 (1999).
${ }^{10}$ H. Hennig, J. Breidbach, and L. S. Cederbaum, "Electron Correlation as the Driving Force for Charge Transfer: Charge Migration Following Ionization in N-Methyl Acetamide," J. Phys. Chem. A 109, 409-414 (2005).

${ }^{11} \mathrm{~J}$. Breidbach and L. S. Cederbaum, "Universal attosecond response to the removal of an electron." Phys. Rev. Lett. 94, 033901 (2005).

${ }^{12}$ A. I. Kuleff and L. S. Cederbaum, "Ultrafast correlation-driven electron dynamics," J. Phys. B: At. Mol. Opt. Phys. 47, 124002 (2014).

${ }^{13}$ A. I. Kuleff, N. V. Kryzhevoi, M. Pernpointner, and L. S. Cederbaum, "Core Ionization Initiates Subfemtosecond Charge Migration in the Valence Shell of Molecules," Phys. Rev. Lett. 117, 093002 (2016).

${ }^{14}$ M. Wormit, D. R. Rehn, P. H. P. Harbach, J. Wenzel, C. M. Krauter, E. Epifanovsky, and A. Dreuw, "Investigating excited electronic states using the algebraic diagrammatic construction (ADC) approach of the polarisation propagator," Mol. Phys. 112, 774-784 (2014).

${ }^{15} \mathrm{~A}$. Dreuw and M. Wormit, "The algebraic diagrammatic construction scheme for the polarization propagator for the calculation of excited states," WIREs Comput Mol Sci 5, 82-95 (2014).

${ }^{16}$ A. I. Kuleff and L. S. Cederbaum, "Charge migration in different conformers of glycine: The role of nuclear geometry," Chem. Phys. 338, 320-328 (2007).

${ }^{17}$ S. Lünnemann, A. I. Kuleff, and L. S. Cederbaum, "Ultrafast charge migration in 2-phenylethyl-N,N-dimethylamine," Chem. Phys. Lett. 450, 232 235 (2008).

${ }^{18}$ S. Lünnemann, A. I. Kuleff, and L. S. Cederbaum, "Charge migration following ionization in systems with chromophore-donor and amine-acceptor sites." J. Chem. Phys. 129, 104305 (2008).

${ }^{19}$ A. I. Kuleff and A. Dreuw, "Theoretical description of charge migration with a single Slater-determinant and beyond.” J. Chem. Phys. 130, 034102 (2009).

${ }^{20}$ S. Lünnemann, A. I. Kuleff, and L. S. Cederbaum, "Ultrafast electron dynamics following outer-valence ionization: the impact of low-lying relaxation satellite states." J. Chem. Phys. 130, 154305 (2009).

${ }^{21}$ A. I. Kuleff, S. Lünnemann, and L. S. Cederbaum, "Ultrafast Charge Migration Following Valence Ionization of 4-Methylphenol: Jumping over the Aromatic Ring †,” J. Phys. Chem. A 114, 8676-8679 (2010).

${ }^{22}$ V. Despré, A. Marciniak, V. Loriot, M. C. E. Galbraith, A. Rouzée, M. J. J. Vrakking, F. Lépine, and A. I. Kuleff, "Attosecond Hole Migration in Benzene Molecules Surviving Nuclear Motion.” J. Phys. Chem. Lett. 6, 426431 (2015).

${ }^{23}$ N. V. Golubev, V. Despré, and A. I. Kuleff, "Quantum control with smoothly varying pulses: general theory and application to charge migration," J. Mod. Opt. 64, 1031-1041 (2017).

${ }^{24}$ K. Lopata and N. Govind, "Modeling Fast Electron Dynamics with RealTime Time-Dependent Density Functional Theory: Application to Small Molecules and Chromophores," J. Chem. Theor. Comput. 7, 1344-1355 (2011).

${ }^{25}$ K. Lopata, B. E. Van Kuiken, M. Khalil, and N. Govind, "Linear-Response and Real-Time Time-Dependent Density Functional Theory Studies of Core-Level Near-Edge X-Ray Absorption,” J. Chem. Theor. Comput. 8, 3284-3292 (2012).

${ }^{26}$ A. Bruner, S. Hernandez, F. Mauger, P. M. Abanador, D. J. LaMaster, M. B. Gaarde, K. J. Schafer, and K. Lopata, "Attosecond Charge Migration with TDDFT: Accurate Dynamics from a Well-Defined Initial State," J. Phys. Chem. Lett. 8, 3991-3996 (2017).

${ }^{27}$ L.-H. Frahm and D. Pfannkuche, "Ultrafast ab Initio Quantum Chemistry Using Matrix Product States.” J. Chem. Theor. Comput. 15, 2154-2165 (2019).

${ }^{28}$ A. Baiardi and M. Reiher, "Large-Scale Quantum Dynamics with Matrix Product States.” J. Chem. Theor. Comput. 15, 3481-3498 (2019).

${ }^{29}$ G. K.-L. Chan and M. Head-Gordon, "Highly correlated calculations with a polynomial cost algorithm: A study of the density matrix renormalization group," 116, 4462-4476 (2002).

${ }^{30}$ J. Ren, Z. Shuai, and G. Kin-Lic Chan, "Time-Dependent Density Matrix Renormalization Group Algorithms for Nearly Exact Absorption and Fluorescence Spectra of Molecular Aggregates at Both Zero and Finite Temperature," J. Chem. Theor. Comput. 14, 5027-5039 (2018).

${ }^{31}$ J. B. Schriber and F. A. Evangelista, "Communication: An adaptive configuration interaction approach for strongly correlated electrons with tunable accuracy," J. Chem. Phys. 144, 161106 (2016).

${ }^{32}$ J. B. Schriber and F. A. Evangelista, "Adaptive Configuration Interaction 
for Computing Challenging Electronic Excited States with Tunable Accuracy." J. Chem. Theor. Comput. 13, 5354-5366 (2017).

${ }^{33}$ J. B. Schriber, K. P. Hannon, C. Li, and F. A. Evangelista, "A Combined Selected Configuration Interaction and Many-Body Treatment of Static and Dynamical Correlation in Oligoacenes." J. Chem. Theor. Comput. 14, 6295-6305 (2018).

${ }^{34} \mathrm{Z}$. Gershgorn and I. Shavitt, "An application of perturbation theory ideas in configuration interaction calculations," Int. J. Quant. Chem. 2, 751-759 (1968).

${ }^{35}$ A. Pipano and I. Shavitt, "Convergence studies in configuration interaction calculations," Int. J. Quant. Chem. 2, 741-749 (1968).

${ }^{36}$ C. F. Bender and E. R. Davidson, "Studies in Configuration Interaction: The First-Row Diatomic Hydrides," Phys. Rev. 183, 23-30 (1969).

${ }^{37}$ J. L. Whitten and M. Hackmeyer, "Configuration Interaction Studies of Ground and Excited States of Polyatomic Molecules. I. The CI Formulation and Studies of Formaldehyde," J. Chem. Phys. 51, 5584-5596 (1969).

${ }^{38}$ B. Huron, J. P. Malrieu, and P. Rancurel, "Iterative perturbation calculations of ground and excited state energies from multiconfigurational zerothorder wavefunctions," J. Chem. Phys. 58, 5745-5759 (1973).

${ }^{39}$ R. J. Buenker and S. D. Peyerimhoff, "Individualized configuration selection in CI calculations with subsequent energy extrapolation," Theoret. Chim. Acta 35, 33-58.

${ }^{40}$ R. J. Buenker and S. D. Peyerimhoff, "Energy extrapolation in CI calculations," Theoret. Chim. Acta 39, 217-228 (1975).

${ }^{41}$ S. Evangelisti, J.-P. Daudey, and J. P. Malrieu, "Convergence of an improved CIPSI algorithm,” Chem. Phys. 75, 91-102 (1983).

${ }^{42} \mathrm{R}$. Cimiraglia, "Second order perturbation correction to CI energies by use of diagrammatic techniques: An improvement to the CIPSI algorithm," J. Chem. Phys. 83, 1746-1749 (1985).

${ }^{43}$ R. J. Harrison, "Approximating full configuration interaction with selected configuration interaction and perturbation theory," J. Chem. Phys. 94, 5021-5031 (1991).

${ }^{44}$ M. Hanrath and B. Engels, "New algorithms for an individually selecting MR-CI program," Chem. Phys. (1997).

${ }^{45}$ P. Stampfuß and W. Wenzel, "Improved implementation and application of the individually selecting configuration interaction method," J. Chem. Phys. 122, 024110 (2005).

${ }^{46}$ L. Bytautas and K. Ruedenberg, "A priori identification of configurational deadwood," Chem. Phys. 356, 64-75 (2009).

${ }^{47}$ J. C. Greer, "Estimating full configuration interaction limits from a Monte Carlo selection of the expansion space," J. Chem. Phys. 103, 1821-1828 (1995).

${ }^{48}$ J. C. Greer, "Monte Carlo Configuration Interaction," J. Comput. Phys. 146, 181-202 (1998).

${ }^{49}$ J. P. Coe and M. J. Paterson, "Development of Monte Carlo configuration interaction: natural orbitals and second-order perturbation theory," J. Chem. Phys. 137, 204108 (2012).

${ }^{50}$ J. P. Coe and M. J. Paterson, "State-averaged Monte Carlo configuration interaction applied to electronically excited states," J. Chem. Phys. 139, 154103 (2013).

${ }^{51}$ P. J. Knowles, "Compressive sampling in configuration interaction wavefunctions," Mol. Phys. 113, 1655-1660 (2015).

${ }^{52}$ J. R. McClean and A. Aspuru-Guzik, "Compact wavefunctions from compressed imaginary time evolution," RSC Adv. 5, 102277-102283 (2015).

${ }^{53}$ C. F. Bunge, "Selected configuration interaction with truncation energy error and application to the Ne atom." J. Chem. Phys. 125, 014107 (2006).

${ }^{54} \mathrm{~F}$. A. Evangelista, "Adaptive multiconfigurational wave functions," 140, 054109 (2014).

${ }^{55}$ N. M. Tubman, J. Lee, T. Y. Takeshita, M. Head-Gordon, and K. B. Whaley, "A deterministic alternative to the full configuration interaction quantum Monte Carlo method," J. Chem. Phys. 145, 044112 (2016).

${ }^{56}$ Y. Ohtsuka and J.-y. Hasegawa, "Selected configuration interaction method using sampled first-order corrections to wave functions." J. Chem. Phys. 147, 034102 (2017).

${ }^{57}$ E. Giner, A. Scemama, and M. Caffarel, "Using perturbatively selected configuration interaction in quantum Monte Carlo calculations," Can. J. Chem. 91, 879-885 (2013).

${ }^{58}$ E. Giner, R. Assaraf, and J. Toulouse, "Quantum Monte Carlo with reoptimised perturbatively selected configuration-interaction wave functions," Mol. Phys. 114, 910-920 (2016).
${ }^{59}$ A. A. Holmes, N. M. Tubman, and C. J. Umrigar, "Heat-Bath Configuration Interaction: An Efficient Selected Configuration Interaction Algorithm Inspired by Heat-Bath Sampling." J. Chem. Theor. Comput. 12, 3674-3680 (2016).

${ }^{60}$ S. Sharma, A. A. Holmes, G. Jeanmairet, A. Alavi, and C. J. Umrigar, "Semistochastic heat-bath configuration interaction method: Selected configuration interaction with semistochastic perturbation theory." J. Chem. Theor. Comput. 13, 1595-1604 (2017).

${ }^{61}$ A. A. Holmes, C. J. Umrigar, and S. Sharma, "Excited states using semistochastic heat-bath configuration interaction.” J. Chem. Phys. 147, 164111 (2017).

${ }^{62}$ J. Li, M. Otten, A. A. Holmes, S. Sharma, and C. J. Umrigar, "Fast semistochastic heat-bath configuration interaction." J. Chem. Phys. 149, 214110 (2018).

${ }^{63} \mathrm{~S}$. Klinkusch, P. Saalfrank, and T. Klamroth, "Laser-induced electron dynamics including photoionization: A heuristic model within timedependent configuration interaction theory," J. Chem. Phys. 131, 114304 (2009).

${ }^{64} \mathrm{D}$. Hochstuhl and M. Bonitz, "Time-dependent restricted-active-space configuration-interaction method for the photoionization of many-electron atoms," Phys. Rev. A 86, 053424 (2012).

${ }^{65} \mathrm{~S}$. Bauch, L. K. Sørensen, and L. B. Madsen, "Time-dependent generalized-active-space configuration-interaction approach to photoionization dynamics of atoms and molecules," Phys. Rev. A 90, 062508 (2014).

${ }^{66} \mathrm{~J}$. J. Goings, P. J. Lestrange, and X. Li, "Real-time time-dependent electronic structure theory," WIREs Comput Mol Sci 8, e1341 (2017).

${ }^{67}$ W.-T. Peng, B. S. Fales, and B. G. Levine, "Simulating Electron Dynamics of Complex Molecules with Time-Dependent Complete Active Space Configuration Interaction," J. Chem. Theor. Comput. 14, 4129-4138 (2018).

${ }^{68}$ H. Liu, A. J. Jenkins, A. Wildman, M. J. Frisch, F. Lipparini, B. Mennucci, and X. Li, "Time-Dependent Complete Active Space Embedded in a Polarizable Force Field," J. Chem. Theor. Comput. 15, 1633-1641 (2019).

${ }^{69}$ K. C. Kulander, "Time-dependent Hartree-Fock theory of multiphoton ionization: Helium," Phys. Rev. A 36, 2726-2738 (1987).

${ }^{70}$ X. Li, S. M. Smith, A. N. Markevitch, D. A. Romanov, R. J. Levis, and H. B. Schlegel, "A time-dependent Hartree-Fock approach for studying the electronic optical response of molecules in intense fields," Phys. Chem. Chem. Phys. 7, 233-239 (2005).

${ }^{71}$ N. Rohringer, A. Gordon, and R. Santra, "Configuration-interaction-based time-dependent orbital approach for ab initio treatment of electronic dynamics in a strong optical laser field," Phys. Rev. A 74, 043420 (2006).

${ }^{72}$ H. B. Schlegel, S. M. Smith, and X. Li, "Electronic optical response of molecules in intense fields: Comparison of TD-HF, TD-CIS, and TDCIS(D) approaches," J. Chem. Phys. 126, 244110 (2007).

${ }^{73}$ P. Krause, T. Klamroth, and P. Saalfrank, "Molecular response properties from explicitly time-dependent configuration interaction methods," J. Chem. Phys. 127, 034107 (2007).

${ }^{74}$ P. Krause, J. A. Sonk, and H. B. Schlegel, "Strong field ionization rates simulated with time-dependent configuration interaction and an absorbing potential," J. Chem. Phys. 140, 174113 (2014).

${ }^{75}$ T. Sato and K. L. Ishikawa, "Time-dependent multiconfiguration selfconsistent-field method based on the occupation-restricted multiple-activespace model for multielectron dynamics in intense laser fields," Phys. Rev. A 91, 023417 (2015).

${ }^{76}$ I. S. Ulusoy, Z. Stewart, and A. K. Wilson, "The role of the CI expansion length in time-dependent studies." J. Chem. Phys. 148, 014107 (2018).

${ }^{77}$ H. D. Meyer, U. Manthe, and L. S. Cederbaum, "The multi-configurational time-dependent Hartree approach," Chem. Phys. Lett. 165, 73-78 (1990).

${ }^{78}$ M. Nest, T. Klamroth, and P. Saalfrank, "The multiconfiguration timedependent Hartree-Fock method for quantum chemical calculations." J. Chem. Phys. 122, 124102 (2005).

${ }^{79}$ R. P. Miranda, A. J. Fisher, L. Stella, and A. P. Horsfield, "A multiconfigurational time-dependent Hartree-Fock method for excited electronic states. I. General formalism and application to open-shell states," J. Chem. Phys. 134, 244101 (2011)

${ }^{80} \mathrm{~J}$. Theilhaber, "Ab initio simulations of sodium using time-dependent density-functional theory." Phys. Rev., B Condens. Matter 46, 1299013003 (1992).

${ }^{81}$ K. Yabana and G. F. Bertsch, "Time-dependent local-density approximation in real time," Phys. Rev., B Condens. Matter 54, 4484-4487 (1996). 
${ }^{82}$ R. Baer and R. Gould, "A method for ab initio nonlinear electron-density evolution,” J. Chem. Phys. 114, 3385-3392 (2001).

${ }^{83}$ E. Dalgaard and H. J. Monkhorst, "Some aspects of the time-dependent coupled-cluster approach to dynamic response functions," Phys. Rev. A 28 , 1217-1222 (1983).

${ }^{84}$ C. Huber and T. Klamroth, "Explicitly time-dependent coupled cluster singles doubles calculations of laser-driven many-electron dynamics." J. Chem. Phys. 134, 054113 (2011).

${ }^{85} \mathrm{~S}$. Kvaal, "Ab initio quantum dynamics using coupled-cluster," J. Chem. Phys. 136, 194109 (2012).

${ }^{86}$ D. R. Nascimento and A. E. DePrince, "Linear Absorption Spectra from Explicitly Time-Dependent Equation-of-Motion Coupled-Cluster Theory." J. Chem. Theor. Comput. 12, 5834-5840 (2016).

${ }^{87}$ D. R. Nascimento and A. E. DePrince III, "Simulation of Near-Edge Xray Absorption Fine Structure with Time-Dependent Equation-of-Motion Coupled-Cluster Theory," J. Phys. Chem. Lett. 8, 2951-2957 (2017).

${ }^{88} \mathrm{H}$. Eshuis and T. Van Voorhis, "The influence of initial conditions on charge transfer dynamics." Phys. Chem. Chem. Phys. 11, 10293-10298 (2009).

${ }^{89} \mathrm{~N}$. T. Maitra and K. Burke, "Demonstration of initial-state dependence in time-dependent density-functional theory," Phys. Rev. A 63, 042501 (2001).
${ }^{90}$ W. H. Press, S. A. Teukolsky, W. T. Vetterling, and B. P. Flannery, "Numerical recipes 3rd edition: The art of scientific computing," (2007).

${ }^{91}$ A. Gómez Pueyo, M. A. L. Marques, A. Rubio, and A. Castro, "Propagators for the Time-Dependent Kohn-Sham Equations: Multistep, Runge-Kutta, Exponential Runge-Kutta, and Commutator Free Magnus Methods," J. Chem. Theor. Comput. 14, 3040-3052 (2018).

${ }^{92}$ R. M. Parrish, L. A. Burns, D. G. A. Smith, A. C. Simmonett, A. E. DePrince, E. G. Hohenstein, U. Bozkaya, A. Y. Sokolov, R. Di Remigio, R. M. Richard, J. F. Gonthier, A. M. James, H. R. McAlexander, A. Kumar, M. Saitow, X. Wang, B. P. Pritchard, P. Verma, H. F. Schaefer, K. Patkowski, R. A. King, E. F. Valeev, F. A. Evangelista, J. M. Turney, T. D. Crawford, and C. D. Sherrill, "Psi4 1.1: An Open-Source Electronic Structure Program Emphasizing Automation, Advanced Libraries, and Interoperability." J. Chem. Theor. Comput. 13, 3185-3197 (2017).

${ }^{93} \mathrm{~F}$. Weigend, "A fully direct RI-HF algorithm: Implementation, optimised auxiliary basis sets, demonstration of accuracy and efficiency," Phys. Chem. Chem. Phys. 4, 4285-4291 (2002).

${ }^{94}$ J. P. Perdew, K. Burke, and M. Ernzerhof, "Generalized Gradient Approximation Made Simple," Phys. Rev. Lett. 77, 3865-3868 (1996).

${ }^{95} \mathrm{~F}$. Weigend and R. Ahlrichs, "Balanced basis sets of split valence, triple zeta valence and quadruple zeta valence quality for $\mathrm{H}$ to $\mathrm{Rn}$ : Design and assessment of accuracy.” Phys. Chem. Chem. Phys. 7, 3297-3305 (2005). 